\title{
Assessment of Resistance in Lettuce (Lactuca sativa L.) to Mycelial and Ascospore Infection by Sclerotinia minor Jagger and $S$. sclerotiorum (Lib.) de Bary
}

\author{
Ryan J. Hayes ${ }^{1}$ \\ USDA-ARS, Crop Improvement and Protection Unit, 1636 E. Alisal Street, \\ Salinas, CA 93905
}

\author{
Bo Ming Wu \\ Oregon State University, Department of Botany and Plant Pathology, \\ Central Oregon Agricultural Research Center, 850 NW Dogwood Lane, \\ Madras, OR 97741
}

Barry M. Pryor

Division of Plant Pathology and Microbiology, University of Arizona, 1140 E. South Campus Drive, Tucson, AZ 85721

Periasamy Chitrampalam and Krishna V. Subbarao

Department of Plant Pathology, University of California, Davis, clo U.S. Agricultural Research Station, 1636 E. Alisal, Salinas, CA 93905

Additional index words. genetic variation, breeding, cultivars, disease resistance, regression, bolting

\begin{abstract}
Lettuce drop caused by Sclerotinia spp. is an economically important disease of lettuce (Lactuca sativa $L_{\text {.) }}$, and cultivars with resistance to mycelial infection by Sclerotinia sclerotiorum (Lib.) de Bary and $S$. minor Jagger as well as to $S$. sclerotiorum ascospores are needed. Assessing resistance in field experiments can be complicated by fast bolting or small stature lettuce lines that may escape rather than resist the pathogens. Therefore, methods to select resistant lines from morphologically variable populations are needed. We used $\boldsymbol{S}$. sclerotiorum and $\boldsymbol{S}$. minor-infested field experiments, regression analysis, field experiments with artificially high plant densities, and $\boldsymbol{S}$. sclerotiorum ascospore inoculations to identify lettuce lines with resistance to both pathogens. Three replicated experiments in $S$. sclerotiorum-infested fields were conducted in Yuma, AZ, and three replicated experiments in a $S$. minor-infested field were conducted in Salinas, CA, using diverse populations of iceberg, romaine, leaf, butterhead, Latin, oilseed lettuce, and wild relatives of lettuces; and genetic variation for the incidence of lettuce drop from mycelial infections was identified. In two $S$. minor field experiments, a quadratic regression model was developed that related rapid bolting with reduced lettuce drop. Regression residuals were calculated, and eight cultivars or PIs had negative residuals in two independent field experiments, indicating higher resistance than predicted by their rate of bolting. Eruption, a small-statured Latin cultivar, had significantly lower disease levels than susceptible cultivars in experiments with high plant densities, indicating that its small size did not facilitate disease escape. Ascospore inoculations confirmed resistance in 'Eruption' and $L$. virosa SAL012, whereas the oilseed lettuce PI 251246 may have partial resistance to infection. These lines will likely be useful for development of Sclerotinia spp.-resistant lettuce cultivars.
\end{abstract}

Lettuce drop is an economically important disease of lettuce. Complete control has not been achieved through cultural practices

\footnotetext{
Received for publication 2 Oct. 2009. Accepted for publication 19 Jan. 2010.

This research was supported by the California Leafy Greens Research Program and the Arizona Iceberg Lettuce Research Council.

${ }^{1}$ To whom reprint requests should be addressed; e-mail Ryan.Hayes@ars.usda.gov.
}

and fungicide applications, making resistant cultivars a potentially valuable tool in an integrated pest management strategy (Subbarao, 1998). Lettuce drop can be caused by two fungal species, Sclerotinia minor and $S$. sclerotiorum. Although the species are known to coexist in western US lettuce production districts, lettuce drop in the Central Valley of California, the Imperial Valley of California, and the Yuma Valley of Arizona, is predominantly caused by S. sclerotiorum (Patterson and Grogan, 1985; Pratt, 1992).
Sclerotinia minor predominates in the coastal valleys of California. Either species may cause mycelial infections of plant crowns, roots, or senescing lower leaves that originate from soilborne sclerotia, a process that is affected by soil moisture and temperature (Abawi and Grogan, 1979; Bell et al., 1998; Steadman, 1979). The characteristic symptoms of these infections include wilting, collapse of lettuce heads, necrosis, and eventual death of the entire plant (Subbarao, 1998). Under cool, wet field conditions, $S$. sclerotiorum can also produce apothecia that release airborne ascospores that directly infect lettuce leaves (Subbarao, 1998). Although the narrow set of environmental conditions that induce ascospore production are relatively rare in commercial fields, the event is always economically devastating (Subbarao, 1998). S. minor can also produce apothecia in laboratory conditions, although its occurrence in field conditions is essentially nonexistent in the United States (Subbarao, 1998). Both species produce long-lived sclerotia allowing the pathogen to persist in the soil for years (Subbarao, 1998). Lettuce cultivars that are resistant to mycelial infections of both species and ascospore infections of $S$. sclerotiorum would provide the greatest protection to lettuce producers. Although the existence of accessions with resistance to both pathogens and infection routes has not been identified, variation for partial resistance to $S$. minor or $S$. sclerotiorum using inoculations of mycelia, sclerotia, or ascospores has been demonstrated (Chupp and Sherf, 1960; Elia and Piglionica, 1964; Grube and Ryder, 2004; Madjid et al., 1983; Newton and Sequeira, 1972; Subbarao, 1998; Whipps et al., 2002).

In the United States, romaine and icebergtype lettuces are the most widely grown. However, many other types of cultivated lettuce are known and encompass extensive variation for leaf color, texture, shape, growth habit, and the time to initiation of bolting (Ryder, 1999). These types include red and green leaf, butterhead, and the lesser known Latin, Batavia, stem, and oilseed lettuce. Latin-type lettuces form small heads with upright leaves that have a soft oily near butterhead-like texture. Batavia lettuces are similar to iceberg but form loosely filled heads of relatively crisp-textured leaves. Stem lettuce, also called asparagus lettuce, is grown for consumption of the stem or core. Oilseed lettuces are fast-bolting highly branched plants that produce seed with high oil content for extraction. Lettuce has three wild relatives that have been successfully used in lettuce breeding (Lebeda et al., 2009). L. serriola and $L$. saligna are annual species, of which $L$. serriola hybridizes freely with lettuce. $L$. virosa is a biennial species and the most distantly related from lettuce among the three wild species (Koopman et al., 1998, 2001).

Host resistance to Sclerotinia spp. in other crops has been attributed to both plant architectural characteristics that facilitate escape of infection and to physiological resistance. Resistance resulting from escape mechanisms in lettuce (rapid bolting, low leaf area, 
and upright growth habit) are well documented (Grube, 2004; Newton and Sequeira, 1972) and may delay or inhibit disease by promoting soil drying, lifting leaves off the soil surface, or lignifications of stems (Grube, 2004; Grube and Ryder, 2004; Newton and Sequeira, 1972; Steadman, 1979). However, many of these traits are not compatible with the specific plant architectures that define modern commercially viable lettuce types. Physiological resistance to Sclerotinia spp. in lettuce that is independent of early bolting or plant morphology, therefore, is more desirable because it is compatible with a diversity of plant forms. It is likely that physiological resistance to Sclerotinia spp. exists in lettuce. For example, genetic variation for the incidence of lettuce drop within cultivated lettuce types having equivalent plant architecture is known (Grube and Aburomia, 2004). Whipps et al. (2002) concluded from direct application of ascospores on lettuce leaves that resistance in the oilseed lettuce accession PI 251246 acted at the time of initial infection. Partially resistant crisphead breeding lines have been developed by the USDA lettuce breeding program in Salinas, CA (Ryder, 1992), but commercial cultivars with an adequate level of resistance to lettuce drop are not yet available (Grube and Ryder, 2004). Development of methods to assess physiological resistance are needed to facilitate breeding of lettuce drop-resistant cultivars for California and Arizona. This is particularly difficult given the wide genetic variation for characters that may facilitate disease escape in wild Lactuca and cultivated lettuce. Although evaluation methods that directly apply the pathogen to host tissue in a manner that negates differences in morphology have been developed for many Sclerotinia spp.-susceptible crops (Ender and Kelley, 2005; Vuong et al., 2004), these are largely not applicable to lettuce. In lettuce, Whipps et al. (2002) directly applied ascospores to lettuce leaves to identify resistant accessions. Methods to assess physiological resistance in field experiments are especially needed. In late blight of potato, residuals from regression of vine maturity on disease severity evaluated in field experiments was used to identify resistance that was independent of late vine maturity (Bradshaw et al., 2004). Modification of this method for evaluation of resistance to Sclerotinia spp. in lettuce could be useful. The objective of this research was to indentify lettuce germplasms with physiological resistance to disease caused by mycelial infection by $S$. minor and S. sclerotiorum and to ascospore infections by $S$. sclerotiorum that are independent of plant morphology.

\section{Materials and Methods}

Field experiments in Yuma, AZ, and Salinas, CA. In Yuma, AZ, six romaine, nine iceberg, three Batavia, three Latin, three green leaf, one butterhead, and two red leaf cultivars along with one oilseed PI and three $L$. virosa accessions were grown over three winter growing seasons in S. sclerotiorum-infested fields. These experiments are referred to as AZ2004,
AZ2005, and AZ2006 (Table 1). Three experiments in a $S$. minor-infested field were conducted in Salinas, CA, during the fall or spring of 2007 and 2008 and are referred to as CAFall2007, CASpring2008, and CAFall2008 (Table 1). These experiments used 26 romaine, seven iceberg, three Batavia, three Latin, 10 green leaf, and two red leaf cultivars, PIs, or breeding lines plus four oilseed PIs. PI 234204, which was tested in CASpring2008, was originally listed in the USDA, Salinas, CA, germplasm database as a L. sativa but was identified as Sonchus oleraceus L. Despite the finding that PI 234204 was not L. sativa, disease assessments of PI 234204 were nonetheless included in the final analysis. In all field experiments, the susceptible control cultivar Batavia Reine des Glaces (BRG) was included. The common commercial control cultivars Salinas 88 (iceberg) and Green Towers (romaine) were used in all Arizona experiments, whereas 'Salinas' (iceberg) and 'Darkland' (romaine) were used as control cultivars in the California experiments. All experiments were arranged in randomized complete block designs with three blocks, and each cultivar was planted to a single plot/block that was 6.1 $\mathrm{m}$ long with two seed lines on a 1-m wide bed. In Arizona, seed was placed on the soil surface at planting, whereas in California, seed was planted at a depth of $0.6 \mathrm{~cm}$. In both locations, seed was germinated with overhead irrigation and thinned to $0.28 \mathrm{~m}$ between plants within a plot $\approx 4$ weeks after seed sowing. After germination, experiments were irrigated as needed using furrow irrigation in Arizona and overhead sprinklers in California. All experiments were subsequently maintained using standard cultural practices for each region (Ryder, 1999).

Evaluating host resistance at different plant densities. Spring and fall field experiments were conducted in Salinas, CA, to evaluate lettuce drop resistance at different plant densities and are referred to as the spring-spacing and fall-spacing experiments (Table 1). These were run concurrently with the CASpring2008 and CAFall2008 experiments and used the same $S$. minor infestation levels, disease assessment, and crop production methods. 'Batavia Reine des Glaces' (BRG, susceptible control), 'Darkland', 'Salinas' (commercial control cultivars), and 'Eruption', were tested in spring-spacing, whereas 'Gladiator' (susceptible control),

Table 1. Location, Sclerotinia spp. pathogen, wet date, date of first disease assessment and total number of disease assessments for eight replicated field experiments to assess resistance in lettuce to lettuce drop.

\begin{tabular}{lllrrr}
\hline Expt. title & Location & \multicolumn{1}{c}{ Pathogen } & Wet date & $\begin{array}{c}\text { Date of } \\
\text { first disease } \\
\text { assessment }^{z}\end{array}$ & $\begin{array}{c}\text { Number } \\
\text { of disease } \\
\text { assessments }^{\mathrm{y}}\end{array}$ \\
\hline AZ2004 & Yuma, AZ & S. sclerotiorum & 9 Nov. 2004 & 15 Jan. 2005 & 6 \\
AZ2005 & Yuma, AZ & S. sclerotiorum & 17 Nov. 2005 & 2 Feb. 2006 & 7 \\
AZ2006 & Yuma, AZ & S. sclerotiorum & 15 Nov. 2006 & 2 Feb. 2007 & 7 \\
CAFall2007 & Salinas, CA & S. minor & 1 Aug. 2007 & 10 Sept. 2007 & 5 \\
CASpring2008 & Salinas, CA & S. minor & 24 Apr. 2008 & 4 June 2008 & 5 \\
CAFall2008 & Salinas, CA & S. minor & 20 Aug. 2008 & 29 Sept. 2008 & 7 \\
Spring-spacing & Salinas, CA & S. minor & 24 Apr. 2008 & 4 June 2008 & 5 \\
Fall-spacing & Salinas, CA & S. minor & 20 Aug. 2008 & 29 Sept. 2008 & 7 \\
\hline
\end{tabular}

${ }^{\mathrm{z}}$ Date the field was first irrigated to initiate germination.

${ }^{y}$ Disease assessments conducted once every $7 \mathrm{~d}$.
'Darkland', 'Salinas' (commercial control cultivars), and 'Eruption' and 'Little Gem' were tested in fall-spacing. To create differences in plant density, one seed line of each plot on a double seed line bed was thinned to $8,18,28,38$, or $48 \mathrm{~cm}$ between plants within a seed line $\approx 4$ weeks after germination. Spacing between seed lines was not adjustable, which could create confounding effects because some large-diameter cultivars are wide enough to reach canopy closure between seed lines, whereas smaller cultivars will not. To eliminate this effect, the plants in the one remaining seed line were completely removed at thinning. The experiment was laid out as a two-way factorial (cultivars and spacing as factors) randomized complete block design with three blocks.

Sclerotinia spp. soil infestation. Field sites were infested with Sclerotinia spp. before seeding to provide uniform inoculum density. For AZ2004, AZ2005, and AZ2006, S. sclerotiorum isolate BMP1995, originally isolated from infected lettuce in a commercial field in Yuma, AZ, was used as the inoculum and was prepared using the methods of Matheron and Porchas (2003). Inoculum consisting of a barley seed-hyphae-sclerotia mixture $(200 \mathrm{~mL})$ was evenly spread over each $6.1-\mathrm{m}$ long plot and lightly mixed into the soil surface with a metal soil rake. Field sites with no history of lettuce drop were chosen for each of these experiments. A single field site was used for the CAFall2007, CASpring2008, and CAFall2008 experiment, which was fumigated with $420 \mathrm{~kg} \cdot \mathrm{ha}^{-1}$ of Tri-Con 57/43 Methyl-Bromide/Chloropicrin (Trical Inc, Hollister, CA) before the CAFall2007 experiment. Sclerotia of S. minor were laid into the seed line using an earthway seeder (Earthway Products, Inc., Bristol, IN) at a rate of $\approx 360 \mathrm{sclerotia} / \mathrm{m}$ for the CAFall2007 and CASpring2008. The CAFall2008 experiment was planted immediately after CASpring2008 and used naturally produced inoculum from infected plants in CASpring2008 rather than artificial infestation. S. minor isolates (BM001, BM005, BM007, and BM010) used were originally isolated from infected lettuce in commercial fields in Salinas, CA. To produce sclerotia of these isolates, $\approx 250 \mathrm{~g}$ of washed and peeled potatoes cut into 1 - to 2 -cm cubes were placed into $500-\mathrm{mL}$ flasks. Flasks were sealed with foil, autoclaved for $30 \mathrm{~min}$, and 
reautoclaved $24 \mathrm{~h}$ later. After the potato pieces cooled down to room temperature, three to four agar plugs $(0.5 \mathrm{~cm}$ diameter $)$ cut out of $S$. minor cultures were transferred into separate flasks. The flasks were incubated at 23 to $24{ }^{\circ} \mathrm{C}$ and periodically shaken to promote uniform colonization of potato pieces. Approximately 6 weeks later, sclerotia were collected by washing flask contents through a fine mesh sieve and straining out the sclerotia. Sclerotia were then air-dried and stored at room temperature until used.

Disease assessment and statistical analysis of field experiments. Lettuce drop incidence was recorded as the number of wilted, collapsed lettuce heads with white mycelium and developing sclerotia on the plant crown. Incidence was recorded every week starting with the first appearance of the disease and continuing until the iceberg cultivars reach market maturity or 'BRG' (the susceptible control) reached near 100\% mortality (Table 1). The cumulative mortality for the entire experiment was totaled, expressed as a proportion based on the total number of plants/plot before disease onset, and transformed to the arcsine scale for analysis using restricted maximum likelihood in Proc Mixed of SAS (Version 9.2; SAS Institute, Inc., Cary, NC). Line (cultivars, breeding lines, PIs, accessions) and year were treated as fixed effects, and block within year was treated as a random effect. Least square means were requested for each year and year $\times$ line combination. Planned paired comparisons to identify significantly different means $(P<0.05)$ were conducted between each line and the susceptible control ('BRG' in all experiments), between each line and important commercial control cultivars (Green Towers and Salinas 88 in AZ2004, AZ2005, and AZ2006; Darkland and Salinas CAFall2007, CASpring2008, and CAFall2008), and between years within the Arizona location. To compare years within the California location, an arithmetic disease rating (DR) mean was calculated for each experiment. To determine if the differences among CAFall2007, CASpring2008, and CAFall2008 were statistically significant at $P<0.05$, a separate Proc Mixed analysis was also conducted using only the 15 cultivars tested in all 3 years. The spring-spacing and fall-spacing experiments were each analyzed separately and used spacing, line, and spacing $\times$ line as fixed effects and block and interactions with block as random effects. Least square means and 99\% confidence intervals were requested for each line $\times$ spacing treatment. For all experiments, means were backtransformed and reported on tables as DR, which was correlated with the original percent mortality data at $r=0.97(P<$ $0.001)$ or higher in these experiments. Correlations coefficients were calculated between years and locations using the DR data.

The rate of bolting was evaluated in the CAFall2007 and CASpring2008 experiments using the plants remaining at the end of the experiment on a 1 to 6 scale in which $1=$ no bolting, 2 = beginning to bolt, $3=$ advanced bolting, 4 = beginning to flower, $5=$ full flowering, and $6=$ seed set. In some cases, insufficient numbers of healthy plants remained at the end of the experiment, and an accurate evaluation could not be determined. In these cases, no assessment of bolting was recorded. A regression analysis was conducted using the software package ARC (Version 1.06, 2004) with DR as the dependant variable and bolting as the independent variable. ARC is freely available at www.stat. umn.edu/arc/software.html. Linear and quadratic regression models were compared using the approach of Cook and Wiesberg (1999). Regression residual, the difference between the actual DR and the predicted DR for each line, was calculated by the software. Scatterplots with fitted regression curves were drawn using Sigmaplot (Version 11.0; Systat Software Inc., San Jose, CA).

Sclerotinia sclerotiorum ascospore production and collection. Sclerotinia sclerotiorum isolates BS001 and BS014 were collected from infected lettuce plants in the Salinas Valley and used to produce ascospores for inoculations. Sclerotia were prepared first for subsequent use in ascospore production. Sclerotia from each isolate were produced on $250 \mathrm{~g}$ of $1-\mathrm{cm}$ cube potato pieces in $500-\mathrm{mL}$ flasks. The potato pieces were autoclaved twice at $121^{\circ} \mathrm{C}$ within a $24-\mathrm{h}$ interval, inoculated with two or three agar disks from the leading edges of cultures grown on potato dextrose agar, and transferred into each flask. Flasks were incubated at room temperature $\left(20 \pm 3{ }^{\circ} \mathrm{C}\right)$ under ambient light conditions for 3 weeks and then the sclerotia of each isolate were harvested by washing off the potato debris, mycelia, and immature sclerotia. Sclerotia were air-dried and stored at room temperature for later use.

Fifty uniform-sized sclerotia (3.4 to 4.8 $\mathrm{mm}$ ) of each isolate were placed on a $2.54-\mathrm{cm}$ thick and $7.62-\mathrm{cm}$ diameter polyurethane foam block (Foamex Intl., San Leandro, $\mathrm{CA}$; density $=16.82 \mathrm{~kg} \cdot \mathrm{m}^{-3}$, indentation load deflection $\left.=2.39 \mathrm{~kg} \cdot \mathrm{cm}^{-2}\right)$ in a Styrofoam cup $(6 \mathrm{~cm}$ in height and diameters at the bottom and top were 7 and $8.5 \mathrm{~cm}$, respectively) with four $2-\mathrm{mm}$ diameter holes punctured at $1-\mathrm{cm}$ height from the bottom. The cups were covered with $100-\mathrm{mm}$ petri dish lids, placed in a plastic box, and incubated in darkness at 18 to $20{ }^{\circ} \mathrm{C}$. Moisture was maintained by misting the sclerotia with sterilized doubledistilled water twice a week, and excessive water was periodically removed from the plastic boxes. Carpogenic germination of sclerotia was evaluated every 2 to $3 \mathrm{~d}$ after the first appearance of stipes. The sclerotia with visible stipes were moved under fluorescent lights for stipes to expand into apothecia. Ascospores were collected from fully expanded apothecia between $1200 \mathrm{HR}$ and $1400 \mathrm{HR}$ because this was considered the optimal time for maximum ascospore release (Wu and Subbarao, 2008). Ascospores were collected on filter paper discs by vacuum suction, which were then wrapped in aluminum foil and stored at $-20{ }^{\circ} \mathrm{C}$ until used.

Growth room experiment to assess resistance to $\mathrm{S}$. sclerotiorum ascospores.
'Eruption', 'Great Lakes 54', 'BRG' (susceptible control), 'Holborn Standard', 'Gladiator', 'Two Star', 'Salinas', PI 251246, PI 176594, PI 207490, and L. virosa accession SAL012 were assessed for disease development after inoculation with ascospores of $S$. sclerotiorum. The experiment was designed as a randomized complete block with six blocks and four plants/line per block. Seeds were germinated in a growth room in sterile plug mix, transplanted into 470-mL Styrofoam cups containing a pasteurized mix of 3 sand:1 potting soil 4 weeks after sowing, and grown with a $16-\mathrm{h}$ daylength at $24^{\circ} \mathrm{C}$. Plants were inoculated 6 weeks after germination. Ascospore inoculum was prepared by washing the ascospores off the filter paper disc with sterile distilled water and diluting the spore suspension to $1.71 \times 10^{6}$ ascospores $/ \mathrm{mL}$. To enhance spore germination on the lettuce leaf surface, an external sugar source in the form of petals was needed ( $\mathrm{Wu}$ and Subbarao, 2008). Flowers were collected from wild-grown mustard plants, the petals were mixed with the spore suspension, and the mixture was evenly sprayed on the plants until runoff. The plants were incubated in the dark for $8 \mathrm{~h}$ at $24^{\circ} \mathrm{C}$ and then returned to $16-\mathrm{h}$ daylength. No supplemental humidity was added during the course of the experiment. Symptoms appeared 1 week after inoculation, and the number of plants with any symptom [disease incidence (DI)] as well as the number of dead plants was recorded every 2 or $3 \mathrm{~d}$ for $46 \mathrm{~d}$ for a total of 18 assessments. After inoculation, a noninoculated treatment of the susceptible cultivar BRG was interspersed into the experiment solely to ensure that symptoms on inoculated plants were caused by $S$. sclerotiorum. Because it was clear that the symptoms were the result of $S$. sclerotiorum, the noninoculated 'BRG' treatment was removed from the data set before analysis. To examine differences in DI (proportion of plants with any symptom) over the 18 reading dates, the cumulative DI data for each reading date were analyzed as a repeated measures experiment in Proc Mixed of SAS following the guidelines of Littell et al. (2006). Reading date, line, and reading date $\times$ line were treated as fixed effects. Block $\times$ line was used as the SUBJECT effect in the SAS syntax, and an autoregressive covariance structure was used. The proportion of plants that had died by the end of the experiment in each line in each repetition was arcsine-ransformed and analyzed in Proc Mixed of SAS (Version 9.2) using line (cultivars, PIs, and accessions) as fixed effects and block and line $\times$ block as a random effect. Least square means were requested for each line, and planned paired comparisons to identify significantly different means $(P<0.05)$ were conducted between each line and the susceptible control cultivar BRG. Means were backtransformed and reported as disease rating (DR).

\section{Results}

Genetic variation for lettuce drop incidence in a soil-infested field experiment. 
Genetic variation for lettuce drop disease ratings was identified among 28 cultivars or PIs of lettuce and three accessions of $L$. virosa in the $S$. sclerotiorum infested field experiments AZ2004, AZ2005, and AZ2006 in Yuma, AZ (Table 2). In AZ2004, the susceptible control cultivar BRG had a DR of 0.54 , whereas the DR of the commercially important cultivars Green Towers and Salinas 88 were 0.16 and 0.28 , respectively. Only PI $251246(\mathrm{DR}=0.07)$ and $L$. virosa accession SAL012 (DR $=0.07)$ had significantly lower DRs than 'BRG'. In AZ2005, five cultivars (Eruption, Little Gem, Medallion, Two Star, and Winterhaven), PI 251246, and three $L$. virosa accessions with mean DR ranging from 0.01 to 0.20 had significantly less disease than 'BRG' (DR = 0.57). L. virosa accession IVT280 also had significantly less disease than 'Green Towers' (DR $=0.35$ ). No lines had significantly less disease than 'Salinas 88' $(\mathrm{DR}=0.22)$. In the AZ2006 field experiment, 13 lines with DR ranging from 0.02 to 0.28 had significantly lower DR than 'BRG' (DR = 0.61). L. virosa accessions SAL012 (DR = $0.02)$ and IVT1398 (DR $=0.03)$ had DR

Table 2. Mean lettuce drop disease rating of 31 lettuce cultivars, PIs, and L. virosa accessions in Sclerotinia sclerotiorum-infested replicated winter field experiments AZ2004, AZ2005, and AZ2006 located in Yuma, AZ.

\begin{tabular}{|c|c|c|c|}
\hline \multirow[b]{2}{*}{$\operatorname{Line}^{z}$} & \multicolumn{3}{|c|}{ Mean disease rating ${ }^{\mathrm{y}, \mathrm{x}}$} \\
\hline & $\overline{\mathrm{AZ2004}}{ }^{\mathrm{w}}$ & AZ2005 & AZ2006 \\
\hline \multicolumn{4}{|l|}{ Control cultivars } \\
\hline Batavia Reine des Glaces (B, SC) & 0.54 & 0.57 & 0.61 \\
\hline Salinas $88(\mathrm{I})$ & 0.28 & 0.22 & 0.32 \\
\hline Green Towers (R) & 0.16 & 0.35 & 0.30 \\
\hline \multicolumn{4}{|l|}{ Experimental lines } \\
\hline PI $251246(\mathrm{O})$ & $0.07 \mathrm{~b}$ & $0.08 \mathrm{~b}$ & $0.07 \mathrm{~b}$ \\
\hline SAL012 (L. virosa) & $0.07 \mathrm{~b}$ & $0.04 \mathrm{~b}$ & $0.02 \mathrm{~b}, \mathrm{~g}, \mathrm{~s}$ \\
\hline IVT280 (L. virosa) & 0.10 & $0.01 \mathrm{~b}, \mathrm{~g}$ & $0.06 \mathrm{~b}$ \\
\hline Medallion (R) & 0.15 & $0.19 \mathrm{~b}$ & 0.35 \\
\hline $\mathrm{PIC} 714(\mathrm{R})$ & 0.15 & 0.26 & $0.17 \mathrm{~b}$ \\
\hline Gladiator (R) & 0.18 & 0.34 & 0.43 \\
\hline Little Gem (L) & 0.19 & $0.17 \mathrm{~b}$ & $0.17 \mathrm{~b}$ \\
\hline Eruption (L) & 0.23 & $0.08 \mathrm{~b}$ & $0.18 \mathrm{~b}$ \\
\hline Fresh Heart (R) & 0.24 & 0.24 & $0.27 \mathrm{~b}$ \\
\hline IVT1398 (L. virosa) & 0.24 & $0.05 \mathrm{~b}$ & $0.03 \mathrm{~b}, \mathrm{~g}, \mathrm{~s}$ \\
\hline King Henry (R) & 0.25 & 0.32 & 0.50 \\
\hline Pavane (L) & 0.25 & 0.22 & $0.28 \mathrm{~b}$ \\
\hline Holborn Standard (B) & 0.27 & 0.30 & $0.19 \mathrm{~b}$ \\
\hline Syn 352 (I) & 0.30 & 0.48 & 0.29 \\
\hline Grizzly (I) & 0.33 & 0.36 & 0.56 \\
\hline Iceberg (B) & 0.33 & 0.37 & $0.21 \mathrm{~b}$ \\
\hline Desert Storm (I) & 0.37 & 0.44 & 0.31 \\
\hline Winterhaven (I) & 0.37 & $0.20 \mathrm{~b}$ & 0.36 \\
\hline Australian (GL) & 0.41 & 0.24 & 0.30 \\
\hline Vanguard 75 (I) & 0.41 & 0.27 & $0.20 \mathrm{~b}$ \\
\hline Coyote (I) & 0.45 & 0.30 & 0.42 \\
\hline Red Tide (RL) & 0.51 & 0.25 & $0.21 \mathrm{~b}$ \\
\hline Red Fox (RL) & 0.55 & 0.28 & 0.35 \\
\hline Tehama (GL) & 0.59 & 0.27 & 0.33 \\
\hline Margarita $(\mathrm{Bu})$ & 0.60 & 0.34 & 0.45 \\
\hline Great Lakes 54 (I) & 0.61 & 0.30 & 0.35 \\
\hline Imperial 850 (I) & 0.64 & 0.33 & 0.50 \\
\hline Two Star (GL) & 0.73 & $0.17 \mathrm{~b}$ & 0.36 \\
\hline Experiment mean & 0.30 & 0.24 & 0.26 \\
\hline
\end{tabular}

${ }^{\mathrm{z}} \mathrm{I}=$ iceberg; $\mathrm{R}=$ romaine; $\mathrm{GL}=$ green leaf; $\mathrm{RL}=$ red leaf; $\mathrm{L}=$ Latin; $\mathrm{B}=$ Batavia; $\mathrm{O}=$ oilseed; $\mathrm{Bu}=$ butterhead; $\mathrm{SC}=$ susceptible control.

${ }^{y}$ Disease ratings are the backtransformed values from analysis of arcsine transformed proportion mortality data.

${ }^{x}$ Values followed by letters indicate significant less disease at $P<0.05$ using a Tukey adjustment; $\mathrm{b}=$ significantly less than BRG; $g$ = significantly less than Green Towers; s = significantly less than Salinas. ${ }^{w}$ AZ2004, AZ2005, and AZ2006 field experiments conducted in Arizona in 2004, 2005, and 2006, respectively. disease incidence (arithmetic DR mean of 0.81 using all lines tested) followed by CASpring $2008(\mathrm{DR}=0.67)$ and CAFall2008 $(\mathrm{DR}=0.57)($ Table 3$)$. Comparing the three experiments using only the 15 cultivars tested in all three experiments, CAFall2007 had a mean DR of 0.88 ; this was significantly higher $(P<0.05)$ than CAFall2008 $(\mathrm{DR}=$ $0.68)$ but not CASpring2008 (DR $=0.80)$. In CAFall2007, the susceptible control cultivar BRG had a DR of 0.97, whereas the commercially important cultivars Darkland and Salinas had DRs of 0.90 and 0.80 , respectively. Only the early flowering oilseed accessions PI 250020 and PI 251246 and the primitive romaine accession PI 226641 were detected with significantly lower DR than 'BRG'. No cultivars had significantly lower disease ratings than 'Darkland' or 'Salinas'. In CASpring2008, 20 lines had significantly lower DR than 'BRG' $(\mathrm{DR}=0.99)$. In addition, five lines ('Eruption', PI 250020, PI 251246, PI 273579, and PI 491214) with DR ranging from 0.03 to 0.23 had significantly less disease than 'Darkland' (DR = 0.82 ) and 'Salinas' (DR $=0.85$ ). Based on the CAFall2007 and CASpring2008 results, a subset of 20 economically important cultivars and other slow bolting cultivars and PIs with superior disease resistance were tested in CAFall2008. Fifteen lines had significantly less disease than 'BRG' $(\mathrm{DR}=0.98)$, 13 had significantly less disease than 'Darkland' as well ( $\mathrm{DR}=0.93$ ), and six ('Cocarde', 'Eruption', 'Little Gem', PI 167391, PI 171665, PI 177418) had significantly less disease than 'Salinas' $(\mathrm{DR}=0.60)$, 'Darkland', and 'BRG'. The correlation between CAFall2007 and CASpring2008 $(r=0.56)$ and CASpring2008 and CAFall2008 $(r=$ $0.74)$ were significant $(P<0.05)$. The correlation between CAFall2007 and CAFall2008 $(r=0.47)$ approached significance $(P=$ $0.057)$. Regardless, the interaction between lines and experiments was significant $(P<$ 0.001 ), because some lines exhibited large differences in DR across experiments. For example, 'Eruption' had a DR of 0.76 in CAFall2007 but a DR of 0.07 in CAFall2008. PI 273579 had a DR of 0.54 in CAFall2007, whereas in CASpring2008, it had a DR of 0.03 .

Regression analysis of bolting and disease rating. The degree of bolting of each line was measured in CAFall2007 and CASpring2008 to use as a covariate with DR. Bolting means ranged from 1 (nonbolting, or rosette plants) to 4.5 ( 5 = full flowering) in CAFall2007 and from 1 to 4 in CASpring2008 (Table 3). The correlation coefficient between bolting score and disease rating was -0.62 for CASpring2008 and -0.51 for CAFall2007. Both correlations were significant $(P<0.01)$ and indicate that faster bolting was associated with less disease. Linear and quadratic regression models were developed for CAFall2007 and CASpring2008 to relate DR to bolting. For both experiments, the quadratic regression modeled the data well (Fig. 1) and resulted in a significantly lower residual sum of squares 
Table 3. Mean disease rating, mean bolting, and regression residuals for 56 lettuce cultivars, PIs, and wild relatives from fall and spring Sclerotinia minor-infested replicated field experiments in 2007 and 2008 located in Salinas, CA.

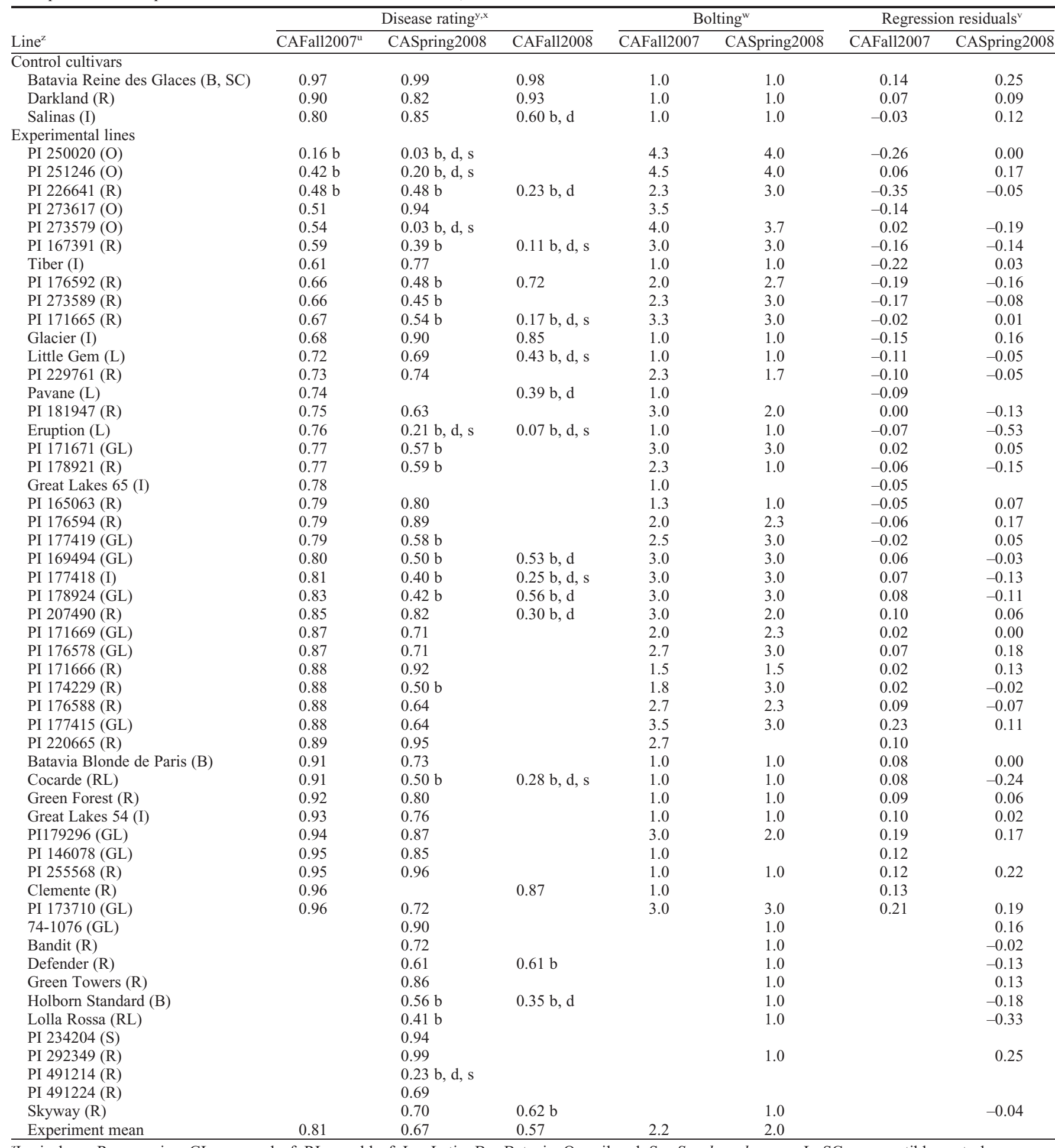

${ }^{\mathrm{z}} \mathrm{I}=$ iceberg; $\mathrm{R}=$ romaine; $\mathrm{GL}=$ green leaf; $\mathrm{RL}=$ red leaf; $\mathrm{L}=$ Latin; $\mathrm{B}=$ Batavia; $\mathrm{O}=$ oilseed; $\mathrm{S}=$ Sonchus oleraceus $\mathrm{L} ; \mathrm{SC}=$ susceptible control.

${ }^{y}$ Disease ratings are the backtransformed values from analysis of arcsine transformed proportion mortality data.

${ }^{x}$ Values followed by letters indicate significant less disease at $P<0.05$ using a Tukey adjustment; $\mathrm{b}=$ significantly less than BRG; $\mathrm{d}=$ significantly less than Darkland; s = significantly less than Salinas.

${ }^{w}$ Bolting, rated $1=$ no bolting by the end of the experiment through $6=$ seed set by the end of the experiment.

${ }^{\mathrm{v}}$ Residuals from quadratic regression of disease rating on bolting.

"CAFall2007, CASpring2008, and CAFall2008 field experiments in California conducting in the spring or fall of 2007 or 2008.

compared with the linear regression (CAFall2007: $F=10.7, P=0.002$ with 1 , 42 df; CASpring2008: $F=15.7, P=0.0003$ with 1, $42 \mathrm{df}$ ). Using the fitted regression line from the quadratic model, residuals were calculated to identify lines that were more resistant than predicted by their rate of bolting, i.e., with DR means below the fitted regression line (Fig. 1). Residuals ranged from -0.35 (PI 226641) to 0.23 (PI 177415) in CAFall2007 and from -0.53 ('Eruption') to 0.25 ('BRG' and PI 292349) for the CASpring2008 experiment (Table 3). The correlation between regression residuals from CAFall2007 and CASpring2008 was 

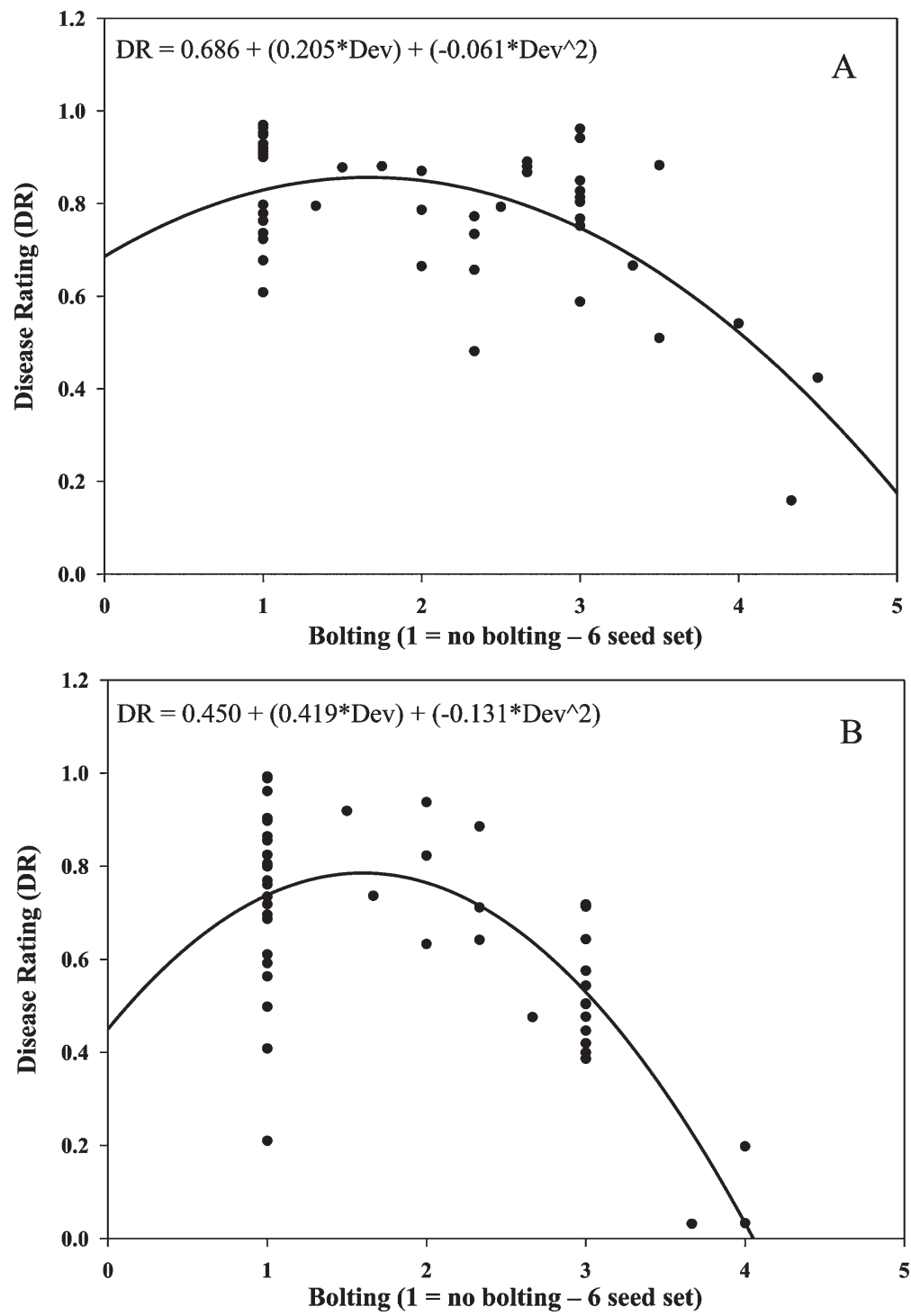

Fig. 1. Quadratic regression of lettuce drop disease rating in a Sclerotinia minor-infested field experiment on bolting using data collected from replicated field experiments of 45 lettuce cultivars, PI, or accessions in CAFall2007 (A) and on 47 lettuce cultivars, PI, or accessions in CASpring2008 (B). Disease rating is calculated from backtransformation of means from arcsine analyzed proportion mortality data. Bolting rated at the end of the experiment (market maturity for 'Salinas') as $1=$ rosetted plant through $6=$ plants with mature seed production.

low $(r=0.33)$ but significant $(P=0.04)$. Furthermore, eight lines ('Eruption', 'Little Gem', PI 167391, PI 176592, PI 178921, PI 226641, PI 229761, and PI 273589) had negative residuals in both experiments, strongly indicating that their resistance is not exclusively based on rapid bolting.

Lettuce drop incidence of cultivars grown under different plant densities. Field experiments indicated that the slow-bolting Latintype cultivars, Eruption and Little Gem, have useful levels of resistance to lettuce drop caused by S. sclerotiorum or S. minor (Tables 2 and 3). However, Latin-type cultivars produce substantially shorter and smaller diameter heads during later growth phases and at market maturity compared with other lettuce types (Fig. 2) that may reduce lettuce drop incidence. Sclerotinia minor-infested field experiments in Salinas, CA, using plant spacing of $8,18,28,38$, and $48 \mathrm{~cm}$ resulted in high plant crowding at the $8-\mathrm{cm}$ spacing (Fig. 2). Furthermore, at the $38-$ and $48-\mathrm{cm}$ spacing, no cultivars achieved row closure during the course of the experiment (data not shown). Despite the differences in plant densities, the effect of spacing on DR was not significant in fall-spacing $(P=0.86)$ or spring-spacing $(P=0.31)$. No significant differences in disease rating between spacing treatments were found within any cultivar (data not shown), and the cultivar $\times$ spacing interaction was also not significant (fallspacing: $P=0.11$; spring-spacing: $P=$ 0.11 ). although the plant spacing treatments applied in these experiments did not affect lettuce drop incidence, significant differences among cultivars were present. Of specific interest is the result that 'Eruption' with the 8 -cm between-plant spacing $(\mathrm{DR}=0.28)$ had significant lower DRs than all other cultivars at all plant spacings in spring-spacing (DR ranging from 0.67 to 0.99 ), except 'Darkland' with $8-(\mathrm{DR}=0.61)$ and $38-\mathrm{cm}$ spacing $(\mathrm{DR}=$ $0.66)$ and 'Salinas' with $28-(\mathrm{DR}=0.54)$ and $48-\mathrm{cm}$ spacing $(\mathrm{DR}=0.55)$. In fall-spacing, 'Eruption' with $8-\mathrm{cm}$ spacing $(\mathrm{DR}=0.07)$ had significantly lower DRs than all cultivar $\times$ spacing treatment combinations (DR ranging from 0.41 to 0.69 ) except 'Little Gem' grown at $38-\mathrm{cm}$ spacing $(\mathrm{DR}=0.33$ ).

Genetic variation for resistance to ascospores. Lesions resulting from ascospore infections appeared on expanded leaf blades $7 \mathrm{~d}$ after inoculation (DAI) and eventually progressed down the petiole to the stem or crown of the plant, at which time the plants experienced systemic wilting, collapse, and finally death. At the time of inoculation, PI 251246, PI 176594, and PI 207490 had already begun to bolt. By the last evaluation, these PIs had reached seed set. DI increased rapidly for all lines except PI 251246 (Fig. $3 \mathrm{~A}$ ). For disease assessments on 7 through 42 DAI, PI 251246 was the only PI with significantly lower DI than 'BRG' (data not shown, $P<0.01)$ and did not reach $100 \%$ DI until 46 DAI. 'Eruption' reached $100 \%$ DI before any other line (18 DAI) and had a significantly higher DI than 'BRG' at 11 and 14 DAI (data not shown, $P<0.01$ ). 'Salinas' had significantly higher DI than 'BRG' at 9 and 11 DAI (data not shown, $P<0.01$ ). The DR of PI 251246, which is based on the final mortality of each line, was not significantly different from 'BRG' (Fig. 3B). Accession SAL012 $(\mathrm{DR}=0.17)$ and 'Eruption' $(\mathrm{DR}=0.25)$ were the only lines with significantly lower DR means than 'BRG' (Fig. 3B).

\section{Discussion}

Genetic variation for resistance to lettuce drop from mycelial infections was identified under field conditions for $S$. sclerotiorum in Yuma, AZ, and for S. minor in Salinas, CA. Managing disease pressure in field experiments is important for routinely generating detectable genetic differences for selection, particularly because all lettuce genotypes will eventually succumb to Sclerotinia spp. infections. For example, heavy disease incidence occurred in CAFall2007, which likely hindered our ability to identify genetic differences for DR for this experiment. Experiments with heavy disease pressure further confirm that all Lactuca accessions tested to date are susceptible to colonization and death by Sclerotinia spp. However, this does not necessarily indicate a lack of genes conferring economically useful levels of partial resistance, because the level of disease found in experiments such as CAFall2007 is far beyond what typically occurs in lettuce production fields. This reality needs to be considered when evaluating the results from lettuce drop field experiments. In the $S$. sclerotiorum-infested field experiments located in Yuma, AZ, L. virosa accessions SAL012, IVT280, and IVT1398, oilseed accession PI 251246, and the Latin cultivars Eruption and Little Gem were the only lines with DR significantly lower than 'BRG' in 
$8 \mathrm{~cm}$ spacing

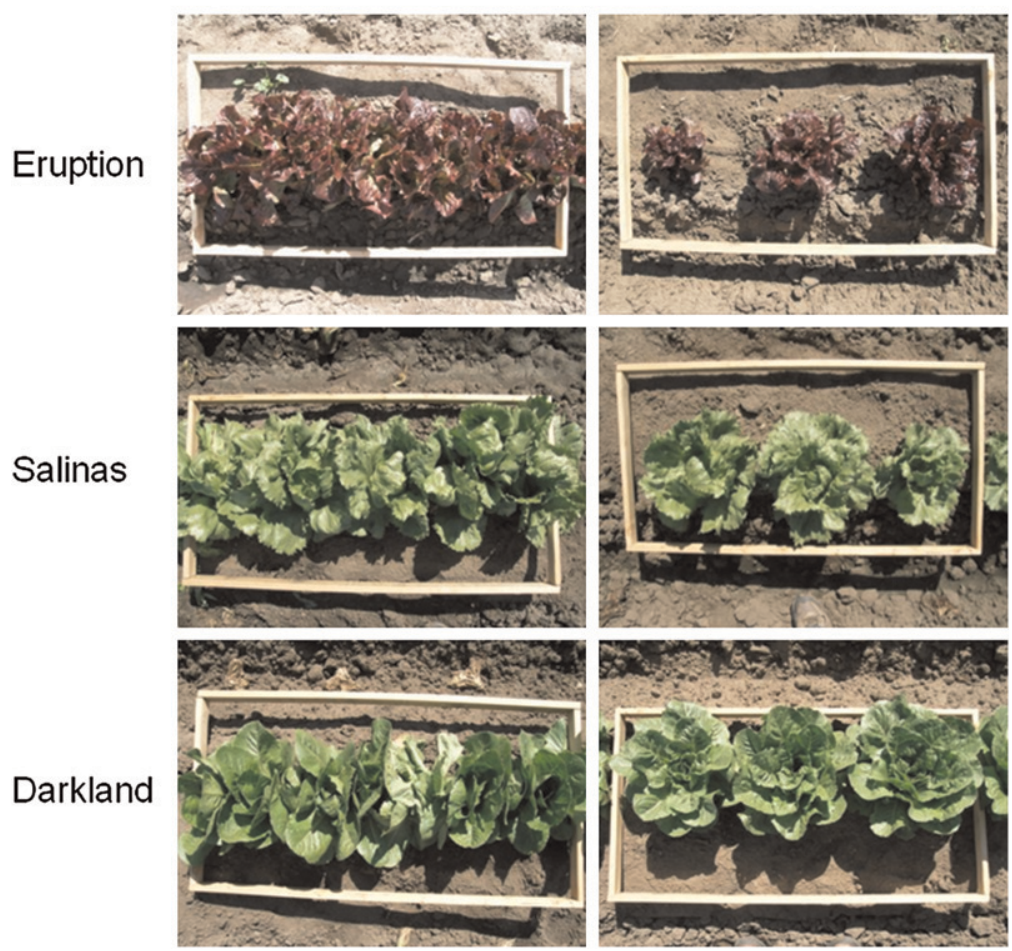

Fig. 2. Cultivars Eruption, Salinas, and Darkland $64 \mathrm{~d}$ after planting in the spring-spacing field experiment in Salinas, CA, with between-plant spacing of $8 \mathrm{~cm}$ and $28 \mathrm{~cm}$. The rectangle in each picture is 104.1 $\mathrm{cm}$ long $\times 54.5 \mathrm{~cm}$ wide.

more than 1 year of testing. Among experiments CAFall2007, CASpring2008, and CAFall2008, 11 lines had significantly lower DR than 'BRG' in more than one experiment. Among the lines tested at both Yuma, AZ, and Salinas, CA, PI 251246, 'Eruption' and 'Little Gem' had at least two field experiments at both locations with DR significantly lower than 'BRG'. Other lines were identified that performed well in one to a few experiments but need further testing to confirm resistance given the line $\times$ environment (year or planting) interactions for resistance that can occur in Sclerotinia-infested field experiments (Tables 1 and 2).

Plant morphology, particularly rapid bolting, is known to influence lettuce drop incidence (Grube, 2004; Grube and Ryder, 2004). Rapid bolting may contribute to resistance through lignifications of stems, which are potentially more difficult for the pathogen to colonize, or through lifting of lower leaves off the soil surface, which promotes drying of the soil near the plant crown (Grube, 2004; Grube and Ryder, 2004). We used regression analysis and subsequent calculation of residuals to identify lines with less disease than predicted by their rate of bolting. Negative residuals calculated from the regression analysis from field experiments may be a useful indicator of physiological resistance. 'Eruption', 'Little Gem', PI 167391, PI 176592, PI 178921, PI 226641, PI 229761, and PI 273589 had negative residuals in two $S$. minor-infested Salinas, CA, field experiments (CAFall2007 and CASpring2008). This approach appears to have promise for identifying new germplasm for use as parents in a lettuce drop breeding program using field experiments in target breeding environments. Several of the lines identified in this research are being incorporated into the USDA lettuce drop resistance breeding program for this reason. The finding that a quadratic model was a better fit to the data than a linear model indicates that the advantage of rapid bolting is most pronounced in the earliest bolting lines. Therefore, it is expected that the relationship between bolting and DI should be weaker in populations of late to moderately early bolting lines. In some cases, moderately early bolting may not confer any advantage for lettuce drop resistance, like in CAFall2007 (Fig. 1). Removal of the lines with bolting means greater than 3.5 from the data set, and recalculation of correlation coefficients between bolting and DR resulted in a reduction in the magnitude of the coefficient. The correlation for CAFall2007 was reduced from -0.51 to $-0.25(P=0.11)$, whereas the coefficient for CASpring2008 was reduced from -0.62 to $-0.46(P=0.0017)$. Consequently, the confounding effect of early flowering on assessments of lettuce drop resistance in field experiments that contain late and moderately early bolting germplasm is likely to be small to negligible. Furthermore, early bolting is a highly negative character and these segregants are discarded without exception in advanced breeding populations. It is unlikely that lettuce breeders would need to routinely conduct an analysis of regressions residuals to select resistant inbred breeding lines because the early bolting lines will have already been discarded. Therefore, this method is most useful for comparing lettuce drop resistance in populations of potential parental lines that exhibit extensive variation for the rate of bolting. The quadratic regression model can also be interpreted to indicate that DR values may increase slightly for lines with bolting values between 1.5 and 2.0. As an alternative analysis, a segmented regression approach (Gbur et al., 1979) can be applied if one assumes that bolting values equal to zero represent biological inactivity and therefore must be the most susceptible stage of plant development. However, it is not clear that the assumption of biological inactivity of rosette plants is a good assumption in the context of lettuce drop resistance. For these reasons, we have opted to use only the quadratic regression model for this population. Regardless, the residuals calculated from either regression model would differ minimally.

The Latin-type cultivars, Little Gem and Eruption, were identified as two cultivars that have resistance that is not dependant on rapid bolting. However, their small size may nonetheless contribute to low disease incidence in field experiments. The spring-spacing and fall-spacing $S$. minor-infested field experiments that grew 'Little Gem', 'Eruption', and four other cultivars at between-plant spacing ranging from 8 to $48 \mathrm{~cm}$ were conducted to force plant crowding and negate any advantage afforded the smaller-sized Latin cultivars. Unexpectedly, we did not find that increasing plant density increased disease incidence in any cultivar. Although a conclusive explanation for this is not known, it may be related to the abnormally high inoculum densities used in these experiments. Regardless, 'Eruption' was more resistant than the remaining cultivars despite the high plant densities, indicating that its small size does not contribute to reduce lettuce drop incidence.

Physiological resistance to lettuce drop in cultivated lettuce that is independent of plant architecture is likely, although its detection in field experiments can be difficult. We used ascospore inoculations to confirm resistance in the slow bolting lines 'Eruption' and SAL012. Although both of these lines quickly developed lesions, the plants survived for long periods despite their infections. Subbarao (1998) described the concept of slow dying for resistance in lettuce to $S$. minor, in which resistance is expressed as a decreased rate of plant death after the plants are infected. It is possible that the results with 'Eruption' and SAL012 are a manifestation of this in the context of $S$. sclerotiorum ascospore infections. It is likely that this resistance is physiologically based. The ascospore inoculation method excludes the confounding effects of plant shading on soil moisture and temperature that can influence mycelial infections. Additionally, growth chamber production resulted in plants with equivalent growth habits but with genetic variation for resistance (Fig. 3A-B). The 


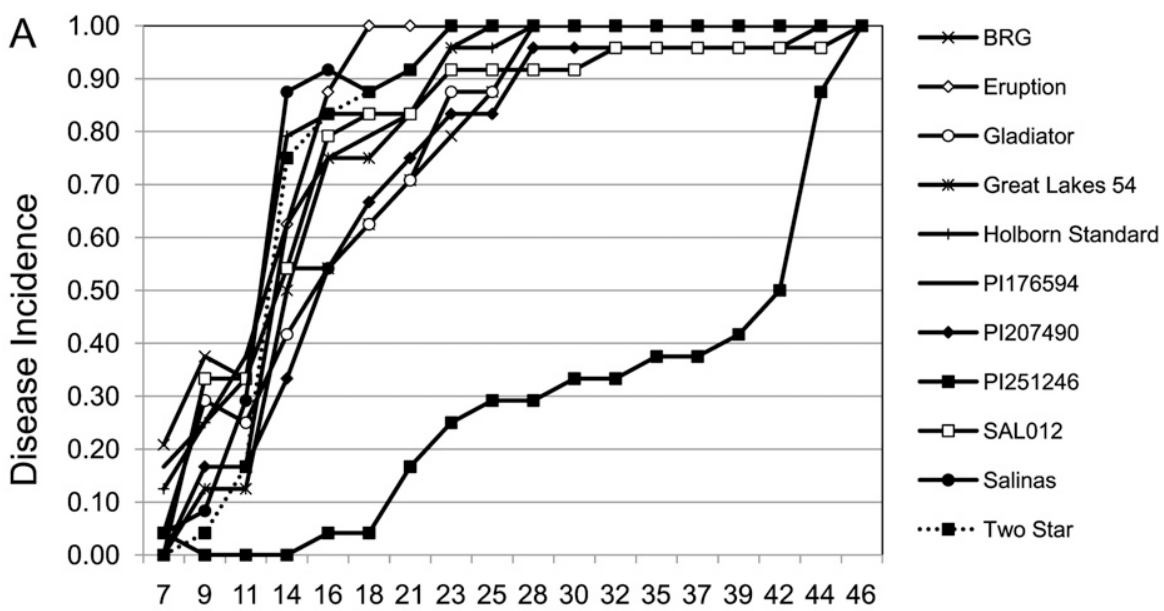

Reading date (days after inoculation)

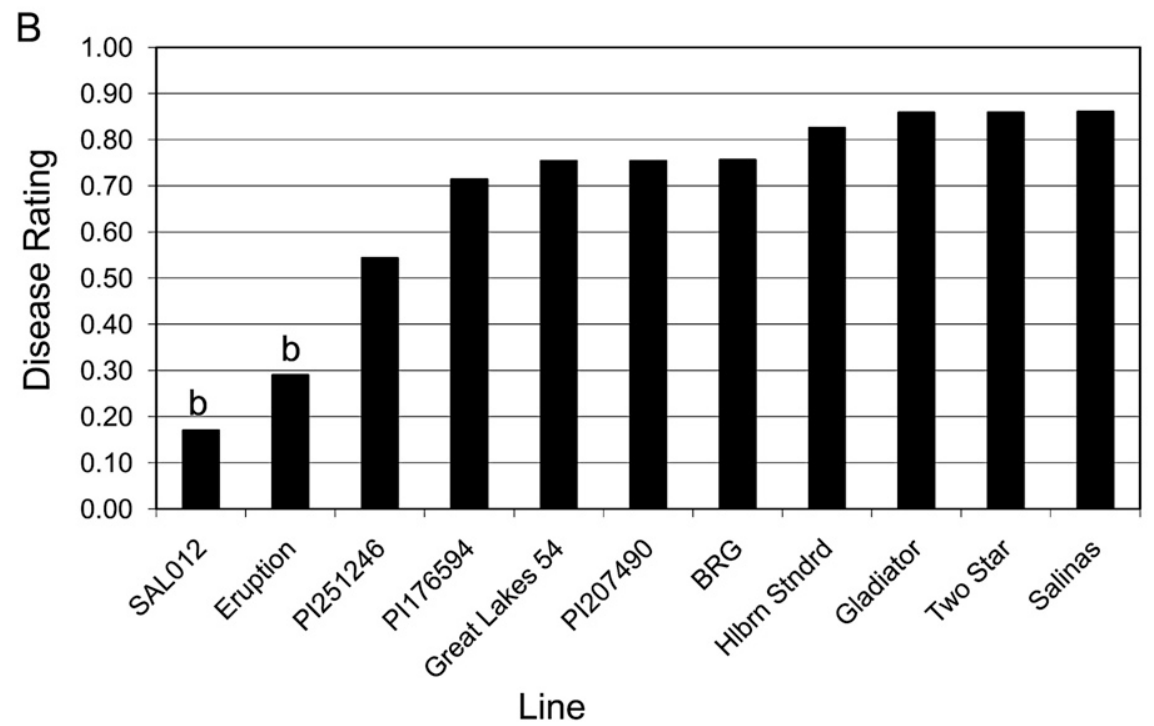

Fig. 3. Inoculation of 11 lettuce cultivars, PIs, and accessions with ascospores of Sclerotinia sclerotiorum $\left(1.71 \times 10^{6} / \mathrm{mL}\right)$ in a growth room experiment with six replications. (A) Chronological change of disease incidence (expressed as the proportion of plants with any disease symptoms) evaluated 18 times every 2 to $3 \mathrm{~d}$ spanning seven to $46 \mathrm{~d}$ after inoculation. (B) Disease rating based on the final mortality (proportion of dead plants) at the end of the experiment ( $46 \mathrm{~d}$ after inoculation). Disease rating is calculated from backtransformation of means from arcsine analyzed proportion mortality data. $\mathrm{BRG}=$ Batavia Reine des Glaces; Hlbrn Stndrd = Holborn Standard; $\mathrm{b}=$ mean disease rating significantly lower than Batavia Reine des Glaces.

rapid bolting oilseed accession PI 251246 had a significantly lower incidence of infected plants compared with 'BRG' for most of the experiment. However, the final DR for PI 251246 was not significantly different from 'BRG'. This would indicate that once these plants are infected, they quickly succumb to the disease. Whipps et al. (2002) came to a similar conclusion, indicating that PI 251246 resisted infection but was no different from the susceptible control for fungal growth in plant tissue once the infection had occurred. It is unclear to what extent early flowering contributes to the ability of PI 251246 to resist infections in this experiment or if this mechanism also operates against mycelial infections of $S$. minor. If resistance to infection does occur with $S$. minor, the finding that PI 251246 had positive residuals calculated from the regres- sion analysis of S. minor-infested field experiments (Table 2) suggests that stem lignifications or other physiological changes resulting from bolting may be associated with this form of resistance. This type of resistance may not be universal to all fast bolting accessions, because PI 176594 and PI 207490 were bolting at the time of ascospore inoculation but were no different for incidence or mortality than 'BRG'.

Development of lettuce drop-resistant romaine and iceberg cultivars is a high priority for the lettuce industry. We have identified lettuce germplasm with partial resistance to $S$. minor and S. sclerotiorum in Yuma, AZ, and Salinas, CA, production environments. Few cultivars were tested in field experiments to both pathogens and at both locations $(\mathrm{n}=6$ for correlation between CAFall2007 and AZ2004, AZ2005, and AZ2006; $\mathrm{n}=7$ for all remaining correlations). However, among the nine correlations calculated between the locations, the coefficients ranged from $r=$ 0.45 to $r=0.94$, and seven were significant $(P<0.05)$. More importantly, for lettuce breeding and genetics research, we have shown that specific lines are resistant ('Eruption', 'Little Gem', PI 251246) or susceptible ('BRG') regardless of the pathogen or experiment location (Tables 1 and 2). Several $L$. virosa accessions demonstrated high levels of resistance, although further analysis is needed to determine what role the slow bolting biennial nature of $L$. virosa plays in resistance. L. virosa has been used successfully in lettuce breeding but requires the use of colchicine-induced chromosome doubling, bridge crossing, and/or embryo rescue (Eenink et al., 1982; Maisonneuve et al., 1995; Thompson and Ryder, 1961). Based on favorable phenotypes observed in families from preliminary crosses, 'Eruption' is likely to be a useful source of resistance to develop resistant romaine cultivars (data not shown). Developing resistant iceberg cultivars with resistance from 'Eruption' would certainly be more challenging as a result of the extremely different morphological characteristics between Latin and iceberg cultivars. An understanding of the inheritance of resistance in 'Eruption' and development of molecular markers linked to quantitative trait loci for resistance could make these breeding projects more efficient.

\section{Literature Cited}

Abawi, G.S. and R.G. Grogan. 1979. Epidemiology of diseases caused by Sclerotinia species. Phytopathology 69:899-904.

Bell, A.A., L. Liu, B. Reidy, R.M. Davis, and K.V. Subbarao. 1998. Mechanisms of subsurface irrigation mediated suppression of lettuce drop caused by Sclerotinia minor. Phytopathology $88: 252-259$.

Bradshaw, J.E., B. Pande, G.J. Bryan, C.A. Hackett, K. McLean, H.E. Stewart, and R. Waugh. 2004 Interval mapping of quantitative trait loci for resistance to late blight [Phytophthora infestans (Mont.) de Bary], height and maturity in a tetraploid population of potato (Solanum tuberosum subsp. tuberosum). Genetics 168: 983-995.

Chupp, C. and A.F. Sherf. 1960. Vegetable diseases and their control. Ronald Press, New York, NY.

Cook, R.D. and S. Wiesberg. 1999. Applied regression including computing and graphics. John Wiley and Sons, New York, NY.

Eenink, A., H.R. Groenwold, and F.L. Dieleman. 1982. Resistance of lettuce (Lactuca) to the leaf aphid Nasanovia ribisnigri. 1. Transfer of resistance from $L$. virosa to $L$. sativa by interspecific crosses and selection of resistant breeding lines. Euphytica 31:291-300.

Elia, M. and V. Piglionica. 1964. Preliminary observations on the resistance of some lettuce cultivars to 'collar rot' caused by Sclerotinia spp. Phytopathol. Mediterr. 3:37-39.

Ender, M. and J.D. Kelley. 2005. Identification of QTL associated with white mold resistance in common bean. Crop Sci. 45:2482-2490.

Gbur, E.E., G.L. Thomas, and F.R. Miller. 1979 Use of segmented regression in the detection of 
the base temperature in heat accumulation models. Agron. J. 71:949-953.

Grube, R. 2004. Genetic analysis of resistance to lettuce drop caused by Sclerotinia minor. Acta Hort. 637:49-55.

Grube, R. and R. Aburomia. 2004. Relationship between plant morphological traits and resistance to Sclerotinia minor in lettuce. HortScience 39:881.

Grube, R. and E.J. Ryder. 2004. Identification of lettuce (Lactuca sativa L.) germplasm with genetic resistance to drop caused by Sclerotinia minor. J. Amer. Soc. Hort. Sci. 129: 70-76.

Koopman, W.J.M., E. Guetta, C.C.M. van de Wiel, B. Vosman, and R.G. Van den Berg. 1998. Phylogenetic relationships among Lactuca (Asteraceae) species and related genera based on ITS-1 DNA sequences. Amer. J. Bot. 85: 1517-1530.

Koopman, W.J.M., M.J. Zevenbergen, and R.G. Van den Berg. 2001. Species relationships in Lactuca s.1. (Lactuceae, Asteraceae) inferred from AFLP fingerprints. Amer. J. Bot. 88: 1881-1887.

Lebeda, A., I. Dolezalova, E. Kristkova, M. Kitner, I. Petrzelova, B. Mieslerova, and A. Novotna. 2009. Wild Lactuca germplasm for breeding: Current status, gaps, and challenges. Euphytica 170:15-34.
Littell, R.C., G.A. Milliken, W.W. Stroup, R.D. Wolfinger, and O. Schabenberger. 2006. SAS for mixed models. SAS Institute Inc., Cary, $\mathrm{NC}$.

Madjid, A., S. Honma, and M.L. Lacy. 1983. A greenhouse method for screening lettuce for resistance to Sclerotinia sclerotiorum. Sci. Hort. 18:201-206.

Maisonneuve, B., M.C. Chupeau, Y. Bellec, and Y. Chupeau. 1995. Sexual and somatic hybridization in the genus Lactuca. Euphytica 85:281285.

Matheron, M.E. and M. Porchas. 2003. The University of Arizona cooperative extension research reports: Vegetable crops, 1997-2003. College of Agriculture and Life Sciences, University of Arizona, Tucson, AZ.

Newton, H.C. and L. Sequeira. 1972. Possible sources of resistance in lettuce to Sclerotinia sclerotiorum. Plant Dis. Rep. 56:875-878.

Patterson, C.L. and R.G. Grogan. 1985. Differences in epidemiology and control of lettuce drop caused by Sclerotinia minor and S. sclerotiorum. Plant Dis. 69:766-770.

Pratt, R.G. 1992. Sclerotinia, p. 74-77. In: Singleton, L.L., J.D. Mihail, and C.M. Rush (eds.). Methods for research on soilborne phytopathogenic fungi. APS Press, St. Paul, MN.

Ryder, E.J. 1992. Notice of release of lettuce germplasm Sclerotinia resistant breeding lines.
U.S. Department of Agriculture, Agricultural Research Service. Washington, DC.

Ryder, E.J. 1999. Lettuce, endive and chicory. CAB International, Wallingford, UK.

Steadman, J.R. 1979. Control of plant disease caused by Sclerotinia species. Phytopathology 69:904-906.

Subbarao, K.V. 1998. Progress towards integrated management of lettuce drop. Plant Dis. 82: 1068-1078.

Thompson, R.C. and E.J. Ryder. 1961. Descriptions and pedigrees of nine varieties of lettuce. Tech. Bull. No. 1244. Agricultural Research Service, U.S. Department of Agriculture, Washington, DC

Vuong, T.D., D.D. Hoffman, B.W. Diers, J.F Miller, J.R. Steadman, and G.L. Hartman. 2004. Evaluation of soybean, dry bean, and sunflower for resistance to Sclerotinia sclerotiorum. Crop Sci. 44:777-783.

Whipps, J.M., S.P. Budge, S. McClement, and D.A. Pink. 2002. A glasshouse cropping method for screening lettuce lines for resistance to Sclerotinia sclerotiorum. Eur. J. Plant Pathol. 108:373-378.

Wu, B.M. and K.V. Subbarao. 2008. Effects of soil temperature, moisture, and burial depths on carpogenic germination of Sclerotinia sclerotiorum and S. minor. Phytopathology 98:11441152. 